2. Asif M, Alvi IA, Rehman SU. Insight into Acinetobacter baumannii: pathogenesis, global resistance, mechanisms of resistance, treatment options, and alternative modalities. Infect Drug Resist 2018; 11: 1249_ 60.

3. Peleg AY, Seifert H, Paterson DL. Acinetobacter baumannii: emergence of a successful pathogen. Clin Microbiol Rev 2008; 21: 538-82.

4. Howard A, O'Donoghue M, Feeney A, et al. Acinetobacter baumannii: an emerging opportunistic pathogen. Virulence 2012; 3: 243-50.

5. Chusri S, Chongsuvivatwong V, Rivera JI, et al. Clinical outcomes of hospital-acquired infection with Acinetobacter nosocomialis and Acinetobacter pittii. Antimicrob Agents Chemother 2014; 58: 4172-9.

6. Munoz-Price LS, Weinstein RA. Acinetobacter infection. $N$ Engl J Med 2018; 358: 1271-81.

7. Cillóniz C, Dominedò C, Torres A. Multidrug resistant Gram-negative bacteria in community-acquired pneumonia. Crit Care 2019; 23 $2371-3$.

8. Guerrero DM, Perez F, Conger NG, et al. Acinetobacter baumanniiassociated skin and soft tissue infections: recognizing a broadening spectrum of disease. Surg Infect (Larchmt) 2010; 11: 49-57.

9. Wong D, Nielsen TB, Bonomo RA, et al. Clinical and pathophysiological overview of Acinetobacter infections: a century of challenges. Clin Microbiol Rev 2016; 30: 409-47.

10. Lee CR, Lee JH, Park M, et al. Biology of Acinetobacter baumannii: pathogenesis, antibiotic resistance mechanisms, and prospective treatment options. Front Cell Infect Microbiol 2017; 7: 55.

11. Akers KS, Chaney C, Barsoumian A, et al. Aminoglycoside resistance and susceptibility testing errors in Acinetobacter baumanniicalcoaceticus complex. J Clin Microbiol 2010; 48: 1132-8.

12. European Committee on Antimicrobial Susceptibility Testing. Breakpoint tables for interpretation of MICs and zone diameters, version 10.0, 2020. Cited 19 Jun 2020. https://www.eucast.org/fileadmin/src/ media/PDFs/EUCAST_files/Breakpoint_tables/v_10.0_Breakpoint_ Tables.pdf.

DOI: https://doi.org/10.1016/j.pathol.2020.10.021

\section{Absence of mecC methicillin- resistant Staphylococcus aureus in a tertiary hospital in Singapore}

Sir,

Methicillin-resistant Staphylococcus aureus (MRSA) are important causes of community acquired and hospital acquired infections. Contact precautions are required to prevent spread from colonised patients to non-colonised patients. Screening for and detection of colonisation is key for infection control purposes. The most common genetic basis for methicillin resistance is the acquisition of the mecA gene coding an altered penicillin-binding protein (PBP)-2A. Divergent $\beta$-lactam resistance conferring mecA homologues such as mecB, mecC, and mecD are also known.

MRSA screening in our hospital is performed using nasal, axilla, and groin swabs inoculated on MRSA Select II agar (Bio-Rad, USA). Although discrepancies between cefoxitin and oxacillin results are more apparent in MRSA harbouring $m e c C$ (cefoxitin resistant, oxacillin susceptible), cefoxitin remains highly reliable for phenotypic detection. ${ }^{2}$ To investigate the molecular epidemiology of MRSA in Singapore, polymerase chain reaction (PCR) screening for mec genes was performed as previously described for $m e c A,{ }^{3}$ mec $C^{4}$ and $m e c B^{5}$ on all included isolates. Between April 2018 and June 2020, a total of 2902 screening isolates from 2482 patients were included. All isolates were confirmed as $S$. aureus with Bruker MALDI Biotyper (Bruker, USA) and were mecApositive $(2902 / 2902 ; 100 \%)$. mecC and $m e c B$ were not detected, confirming $m e c A$ as the dominant mechanism of methicillin-resistance in MRSA in our hospital. A previous meta-analysis indicates $\approx 0.004 \%$ (95\% CI $0.002-0.007)$ mecC prevalence in humans and in animals it is higher at $\approx 0.098 \%$ (95\% CI $0.033-0.174) .{ }^{6}$ We did not detect any $m e c C$ in our clinical setting.

$m e c C$-positive isolates have been found to belong to two predominant lineages, $\mathrm{CC} 130$ and ST425. ${ }^{7}$ Although $\mathrm{mecC}$ MRSA is found from humans and animals, both CC130 and ST425 lineages are adapted to animal hosts suggesting a zoonotic link with acquisition of mecC MRSA. ${ }^{7}$ In Singapore, for geographical reasons, agriculture and farming are not major economic activities. The absence of mecC in our population reflects the limited direct contact with livestock and ruminants. Reports of $m e c C$ in Asia have so far been restricted to single case reports from animals. ${ }^{8,9}$ One isolate from a domestic cat has also been described in Australia. ${ }^{10}$

In summary, mecC remains undetected in Singapore, with scant reports in neighbouring countries. PCR-based commercial assays such as Xpert MRSA NxG (Cepheid, USA), BD MAX StaphSR Assay Kit (Becton Dickinson, USA) and artus MRSA/SA QS-RGQ Kit (Qiagen, Germany) have incorporated mecC detection but this is unlikely to improve the yield of detection in our local population.

Conflicts of interest and sources of funding: The authors state that there are no conflicts of interest to disclose.

\section{Ka Lip Chew ${ }^{1}$, Raymond T. P. Lin ${ }^{1,2}$, Jeanette W. P. Teo}

${ }^{1}$ Department of Laboratory Medicine, National University Hospital, Singapore; ${ }^{2}$ National Public Health Laboratory, National Centre for Infectious Diseases, Singapore

Contact Dr Ka Lip Chew.

E-mail: Ka_lip_chew@nuhs.edu.sg

1. Lakhundi S, Zhang K. Methicillin-resistant Staphylococcus aureus: molecular characterization, evolution, and epidemiology. Clin Microbiol Rev 2018; 31: e00020-18.

2. Kriegeskorte A, Idelevich EA, Schlattmann A, et al. Comparison of different phenotypic approaches to screen and detect mecC-harboring methicillin-resistant Staphylococcus aureus. J Clin Microbiol 2018 56: e00826-17.

3. Reischl U, Linde HJ, Metz M, Leppmeier B, Lehn N. Rapid identification of methicillin-resistant Staphylococcus aureus and simultaneous species confirmation using real-time fluorescence PCR. J Clin Microbiol 2000; 38: 2429-33.

4. Paterson GK, Larsen AR, Robb A, et al. The newly described mecA homologue, mecALGA251, is present in methicillin-resistant Staphylococcus aureus isolates from a diverse range of host species. J Antimicrob Chemother 2012; 67: 2809-13.

5. Becker K, Alen S van, Idelevich EA, et al. Plasmid-encoded transferable mecB-mediated methicillin resistance in Staphylococcus aureus. Emerg Infect Dis 2018; 24: 242.

6. Diaz R, Ramalheira E, Afreixo V, Gago B. Methicillin-resistant Staphylococcus aureus carrying the new mecC gene-a meta-analysis Diagn Microbiol Infect Dis 2016; 84: 135-40.

7. Paterson GK, Harrison EM, Holmes MA. The emergence of mecC methicillin-resistant Staphylococcus aureus. Trends Microbiol 2014; 22: $42-7$

8. Sekizuka T, Niwa $\mathrm{H}$, Kinoshita Y, et al. Identification of a mecA/mecC positive MRSA ST1-t127 isolate from a racehorse in Japan. J Antimicrob Chemother 2020; 75: 292-5.

9. Aklilu E, Chia HY. First mec C and mec A positive livestock-associated methicillin resistant Staphylococcus aureus (mecC MRSA/LA-MRSA ) from dairy cattle in Malaysia. Microorganisms 2020; 8: 147.

10. Worthing KA, Coombs GW, Pang S, et al. Isolation of mecC MRSA in Australia. J Antimicrob Chemother 2016; 71: 2348-9.

DOI: https://doi.org/10.1016/j.pathol.2020.11.006 